\title{
Ultrametric spaces bi-Lipschitz embeddable in $\mathbb{R}^{n}$
}

by

\author{
Kerkko L u o st o (Helsinki)
}

\begin{abstract}
It is proved that if an ultrametric space can be bi-Lipschitz embedded in $\mathbb{R}^{n}$, then its Assouad dimension is less than $n$. Together with a result of Luukkainen and Movahedi-Lankarani, where the converse was shown, this gives a characterization in terms of Assouad dimension of the ultrametric spaces which are bi-Lipschitz embeddable in $\mathbb{R}^{n}$.
\end{abstract}

1. Introduction. Embeddability is a central theme in modern mathematics, common to various different fields such as topology, algebra, functional analysis and set theory. Here the specific notion under consideration is bi-Lipschitz embeddability and the spaces are ultrametric or Euclidean spaces. In this context, the metric dimension introduced by Assouad $[\mathrm{A}]$, or the Assouad dimension, plays a key rôle. In [LM-L, Theorem 3.8], Luukkainen and Movahedi-Lankarani showed that every ultrametric space $(X, d)$ whose Assouad dimension $\operatorname{dim}_{\mathrm{A}}(X, d)$ is strictly less than $n$ is bi-Lipschitz embeddable in $\mathbb{R}^{n}$ (see Definitions 3.1 and 4.1 below). In this paper I prove that the converse also holds, i.e., if an ultrametric space $(X, d)$ can be bi-Lipschitz embedded in $\mathbb{R}^{n}$, then necessarily $\operatorname{dim}_{\mathrm{A}}(X, d)<n$. Movahedi-Lankarani and Wells [M-LW] have independently arrived at a weaker result implying that if $(X, d)$ can be bi-Lipschitz embedded in $\mathbb{R}^{n}$, then the image of $X$ under this embedding has Lebesgue measure zero.

The result of [LM-L] implies that an ultrametric space can be bi-Lipschitz embedded in $\mathbb{R}^{n}$ if and only if its Assouad dimension is finite (see also [A]). According to Semmes [S, Theorem 7.1], this cannot be generalized to arbitrary metric spaces, since, while the necessity is clear, the Heisenberg group with Carnot metric gives a counter-example to the sufficiency. For readers interested in the history of this subject, I recommend the introduction of the paper [LM-L].

1991 Mathematics Subject Classification: Primary 54E40; Secondary 54F45.

This work was partially supported by Emil Aaltonen Foundation. 
Suppose $(X, d)$ is an ultrametric space and $f: X \rightarrow \mathbb{R}^{n}$ a bi-Lipschitz embedding. The proof that $\operatorname{dim}_{A}(X, d)<n$ is organized as follows. In Section 2 , the diameters of finite subsets of $\mathbb{R}^{n}$ are estimated with the aid of a certain fixed linear order on $\mathbb{R}^{n}$. More specifically, given a finite set $Y \subset \mathbb{R}^{n}$ and, for all $y \in Y$, the distance of $y$ from the set of the predecessors of $y$ in $Y$, Proposition 2.2 will give a lower bound for the diameter of $Y$. To motivate this setting, note that $(X, d)$ has a clear-cut structure being an ultrametric space, as for every positive $r$, the open balls with radius $r$ form a partition of $X$. One consequence of this fact is that if we consider a finite set $Z \subset X$ and a linear order $\preceq$ on $Z$, then the set of distances of elements of $Z$ from the sets of their predecessors does not depend on $\preceq$ (compare with the discussion after the proof of Proposition 3.4). Thus the result of Section 2 can easily be used to estimate the size of $f[Z]$ under a bi-Lipschitz mapping $f$.

Section 3 deals with the structural properties of the ultrametric space $(X, d)$. This section introduces a function $\nu_{n}$ defined on all non-empty finite subsets of $X$, which should be regarded as some kind of invariant of the space $(X, d)$. Using the result from Section 2, the function $\nu_{n}$ is related to the sizes of images of finite subsets of $X$. In the next section everything is drawn together and the result $\operatorname{dim}_{\mathrm{A}}(X, d)<n$ is proved. The last section studies the bi-Lipschitz embeddability from a different point of view: special attention is paid to bi-Lipschitz coefficients which are close to 1 . The result is roughly that the smaller the coefficient, the smaller the Assouad dimension of the space to be embedded.

The following notation is used: $\mathbb{N}$ is the set of natural numbers (including zero), $\mathbb{N}^{*}=\mathbb{N} \backslash\{0\}, \mathbb{Z}$ is the set of integers, $\mathbb{R}$ is the set of reals, $\mathbb{R}_{+}=$ $\left[0, \infty\left[=\{r \in \mathbb{R} \mid r \geq 0\}\right.\right.$, and $\mathbb{R}_{+}^{*}=\mathbb{R}_{+} \backslash\{0\}$. The natural number $n$ is identified with the set of its predecessors, i.e., $n=\{0, \ldots, n-1\}$. This settheoretic convention explains notation like $\min _{i \in n} x_{i}=\min \left\{x_{0}, \ldots, x_{n-1}\right\}$ and $I \subset n$. For any set $X$, the power set of $X$ is denoted by $\mathcal{P}(X)$, the set of finite subsets of $X$ by $\mathcal{P}_{\omega}(X)$ and the cardinality of $X$ by $|X|$. We also set $\mathcal{P}_{\omega}^{*}(X)=\mathcal{P}_{\omega}(X) \backslash\{\emptyset\}$ and $\lfloor x\rfloor=\max \{n \in \mathbb{Z} \mid n \leq x\}$. In the course of this paper we shall meet several linear orders. Instead of relying on the context, I have chosen to emphasize the particular linear order used by writing it as a subscript, e.g., as in $\max _{\preceq} A$.

For a metric space $(X, d)$ and a set $Y \subset X$, the diameter of $Y$ is denoted by $\delta(Y)$. If $Y$ is finite and $|Y| \geq 2$, the notation

$$
a(Y)=\min \{d(x, y) \mid x, y \in Y, x \neq y\}
$$

is used. The open ball with centre $x \in X$ and radius $r>0$ is denoted by $B_{d}(x, r)$.

I thank Jouni Luukkainen for introducing me to this subject and for his continued interest in my work. 
2. An estimate for the diameter of a finite subset. Let us fix a linear order $\sqsubseteq$ on the space $\mathbb{R}^{n}$ such that if $\bar{x}=\left(x_{0}, \ldots, x_{n-1}\right) \sqsubseteq \bar{y}=$ $\left(y_{0}, \ldots, y_{n-1}\right)$, then $\sum_{i=0}^{n-1} x_{i} \leq \sum_{i=0}^{n-1} y_{i}$. For the sake of unambiguity, let us agree that for distinct points $\bar{x}=\left(x_{0}, \ldots, x_{n-1}\right), \bar{y}=\left(y_{0}, \ldots, y_{n-1}\right) \in \mathbb{R}^{n}$, we have $\bar{x} \sqsubseteq \bar{y}$ if and only if either $\sum_{i=0}^{n-1} x_{i}<\sum_{i=0}^{n-1} y_{i}$, or $\sum_{i=0}^{n-1} x_{i}=$ $\sum_{i=0}^{n-1} y_{i}$ and $x_{k}<y_{k}$, where $k$ is the least number such that the coordinates $x_{k}$ and $y_{k}$ differ.

Let $A=\left\{\bar{x}_{0}, \ldots, \bar{x}_{k-1}\right\} \subset \mathbb{R}^{n}$, where $k \in \mathbb{N}^{*}$ and the elements of $A$ are enumerated in the increasing order with respect to $\sqsubseteq$. Suppose we are given the following data: For every $l<k-1$ we know the distance of $\bar{x}_{l+1}$ from the set $\left\{\bar{x}_{0}, \ldots, \bar{x}_{l}\right\}$. The objective of this section is to derive a lower bound for $\delta(A)$ using this piece of information. The estimate is obtained by inductively considering for every $l \leq k$ the diameters of the projections of the set $\left\{\bar{x}_{0}, \ldots, \bar{x}_{l-1}\right\}$ onto coordinate axes.

A crucial point here is that the order $\sqsubseteq$ works well with the projections, which is reflected in the fact that, by the compactness of the set $K=\left\{\bar{x}=\left(x_{0}, \ldots, x_{n-1}\right) \in \mathbb{R}^{n}|| \bar{x} \mid=1\right.$ and $\left.\sum_{i=0}^{n-1} x_{i} \geq 0\right\}$ and the continuity of the function $f: \mathbb{R}^{n} \rightarrow \mathbb{R}, f\left(x_{0}, \ldots, x_{n-1}\right)=\max _{i \in n} x_{i}$, there is $\left.\left.\varkappa_{n} \in\right] 0,1\right]$ such that for every unit vector $\bar{x}=\left(x_{0}, \ldots, x_{n-1}\right)$, if the sum of its coordinates is non-negative, then $\max _{i \in n} x_{i} \geq \varkappa_{n}$. In fact, it can be shown by elementary methods that $\varkappa_{1}=1$ and $\varkappa_{n}=1 / \sqrt{n(n-1)}$ for $n \geq 2$ are the best possible choices.

The linear order $\sqsubseteq$ on $\mathbb{R}^{n}$ and the constant $\varkappa_{n}$ remain fixed for the rest of this paper. The derivation of the estimate uses the following lemma of combinatorial nature.

2.1. Lemma. Let $(A, \leq)$ be a finite non-empty linearly ordered set and $\sigma: A \rightarrow \mathbb{R}_{+}$a function such that $\sigma\left(\min _{\leq} A\right)=0$. Suppose that $n \in \mathbb{N}^{*}$ and that $B_{i}: A \rightarrow \mathcal{P}(A), i \in n$, are functions such that for every $a \in A$ the set $\{b \in A \mid b<a\}$ is a disjoint union of the sets $B_{i}(a), i \in n$. Suppose further that functions $f_{i}: A \rightarrow \mathbb{R}_{+}, i \in n$, satisfy the condition $f_{i}(a) \geq f_{i}(b)+\sigma(a)$, for every $i \in n, a \in A$ and $b \in B_{i}(a)$. Then

$$
\max _{a \in A} \max _{i \in n} f_{i}(a) \geq \frac{1}{2} \sqrt[n]{\sum_{a \in A} \sigma(a)^{n}} .
$$

Proof. Let us first split $A$ into classes according to how the sets $B_{i}(a)$ behave. More specifically, let $J_{a}=\left\{i \in n \mid B_{i}(a) \neq \emptyset\right\}$, for every $a \in A$, and let $A_{I}=\left\{a \in A \mid J_{a}=I\right\}$, for $I \subset n$. Note that $A_{\emptyset}=\left\{\min _{\leq} A\right\}$. The sum $S=\sum_{a \in A} \sigma(a)^{n}$ may be arranged as $S=\sum_{I \in \mathcal{I}} \sum_{a \in A_{I}} \sigma(a)^{n}$, where $\mathcal{I}=\left\{I \subset n \mid A_{I} \neq \emptyset\right\}$, whence there is $J \in \mathcal{I}$ such that $\sum_{a \in A_{J}} \sigma(a)^{n} \geq$ $S /|\mathcal{I}| \geq S / 2^{n}$. We may assume $S>0$, which implies $J \neq \emptyset$. Let $m=|J|>0$ 
and $\varrho=\left(\sum_{a \in A_{J}} \sigma(a)^{m}\right)^{1 / m}$; then $\varrho \geq\left(\sum_{a \in A_{J}} \sigma(a)^{n}\right)^{1 / n} \geq 2^{-1} S^{1 / n}$ due to a well-known inequality.

Let $J=\left\{j_{0}, \ldots, j_{m-1}\right\}$, where $j_{0}<\ldots<j_{m-1}$, and for every $a \in A_{J}$ consider the set

$$
Q_{a}=\bigcup_{b \in A_{J}, b \leq a}\left[0, f_{j_{0}}(b)\right] \times \ldots \times\left[0, f_{j_{m-1}}(b)\right] .
$$

Since $A_{J}$ is finite and linearly ordered by $\leq$, we may use induction on $a \in A_{J}$ to prove that $\mu\left(Q_{a}\right) \geq \sum_{b \in A_{J}, b \leq a} \sigma(b)^{m}$, where $\mu$ is the ordinary Lebesgue measure on $\mathbb{R}^{m}$.

1) If $a$ is an element of $A_{J}$, then picking any $b_{k} \in B_{j_{k}}(a)$, for $k \in m$, shows that $f_{j_{k}}(a) \geq f_{j_{k}}\left(b_{k}\right)+\sigma(a) \geq \sigma(a)$. In particular, $\mu\left(Q_{a}\right)=\prod_{k=0}^{m-1} f_{j_{k}}(a) \geq$ $\sigma(a)^{m}=\sum_{b \in A_{J}, b \leq a} \sigma(b)^{m}$ holds for the least element $a$ of $A_{J}$.

2) Suppose then that $a \in A_{J}$ has an immediate predecessor $c$ in $A_{J}$. Let $Q^{*}=\left[f_{j_{0}}(a)-\sigma(a), f_{j_{0}}(a)\right] \times \ldots \times\left[f_{j_{m-1}}(a)-\sigma(a), f_{j_{m-1}}(a)\right]$. Then $Q^{*} \subset Q_{a}$ by the first inequality in the previous paragraph. If $\left(x_{0}, \ldots, x_{m-1}\right) \in Q^{*} \cap Q_{c}$, there is $b \leq c$ such that for all $k \in m, f_{j_{k}}(a)-\sigma(a) \leq x_{k} \leq f_{j_{k}}(b)$. As $b \in \bigcup_{i \in J} B_{i}(a)$, it follows that, for some $k \in m, f_{j_{k}}(a) \geq f_{j_{k}}(b)+\sigma(a)$, which implies $x_{k}=f_{j_{k}}(a)-\sigma(a)$. Hence, $Q^{*} \cap Q_{c} \subset \bigcup_{k=0}^{m-1}\left\{\left(x_{0}, \ldots, x_{m-1}\right) \in\right.$ $\left.\mathbb{R}^{m} \mid x_{k}=f_{j_{k}}(a)-\sigma(a)\right\}$ has measure zero and by the induction hypothesis,

$$
\begin{aligned}
\mu\left(Q_{a}\right) & \geq \mu\left(Q_{c} \cup Q^{*}\right)=\mu\left(Q_{c}\right)+\mu\left(Q^{*}\right) \\
& \geq \sum_{b \in A_{J}, b \leq c} \sigma(b)^{m}+\sigma(a)^{m}=\sum_{b \in A_{J}, b \leq a} \sigma(b)^{m},
\end{aligned}
$$

so the induction claim is proved.

Choosing $a=\max _{\leq} A_{J}$ we have $\mu\left(Q_{a}\right) \geq \sum_{b \in A_{J}} \sigma(b)^{m}=\varrho^{m}$. So $Q_{a}$ is not included in any $m$-cube with the length of an edge less than $\varrho$, which implies that for some $b \in A_{J}, \max _{i \in n} f_{i}(b) \geq \varrho$. Consequently,

$$
\max _{a \in A} \max _{i \in n} f_{i}(a) \geq \varrho \geq \frac{1}{2} S^{1 / n}=\frac{1}{2}\left(\sum_{a \in A} \sigma(a)^{n}\right)^{1 / n}
$$

The preceding lemma easily yields the desired lower bound for the diameter of a given finite set.

2.2. Proposition. Let $A \subset \mathbb{R}^{n}$ be finite and non-empty and let $\sigma$ : $A \rightarrow \mathbb{R}_{+}$,

$$
\sigma(\bar{x})= \begin{cases}0 & \text { for } \bar{x}=\min _{\sqsubseteq} A, \\ \varkappa_{n} \min \{|\bar{y}-\bar{x}| \mid \bar{y} \in A, \bar{y} \sqsubset \bar{x}\} & \text { otherwise. }\end{cases}
$$


Then

$$
\delta(A) \geq \frac{1}{2} \sqrt[n]{\sum_{\bar{x} \in A} \sigma(\bar{x})^{n}}
$$

Proof. For $i \in n$, let $p_{i}: \mathbb{R}^{n} \rightarrow \mathbb{R}, p_{i}\left(x_{0}, \ldots, x_{n-1}\right)=x_{i}$. Suppose $\bar{x}, \bar{y} \in A$ are such that $\bar{x} \sqsubset \bar{y}$. Recall that if $\bar{z} \in \mathbb{R}^{n}$ is a unit vector such that $\sum_{i \in n} p_{i}(\bar{z}) \geq 0$, then $\max _{i \in n} p_{i}(\bar{z}) \geq \varkappa_{n}$. This holds in particular for $\bar{z}=$ $(\bar{y}-\bar{x}) /|\bar{y}-\bar{x}|$, which implies that $\max _{i \in n} p_{i}(\bar{y}-\bar{x})=|\bar{y}-\bar{x}| \max _{i \in n} p_{i}(\bar{z}) \geq$ $\varkappa_{n}|\bar{y}-\bar{x}|$. Denote by $k_{\bar{y}, \bar{x}}$ the least $i \in n$ such that $p_{i}(\bar{y}-\bar{x}) \geq \varkappa_{n}|\bar{y}-\bar{x}|$. For every $i \in n$, let $B_{i}: A \rightarrow \mathcal{P}(A), B_{i}(\bar{y})=\left\{\bar{x} \in A \mid \bar{x} \sqsubset \bar{y}, k_{\bar{y}, \bar{x}}=i\right\}$ and $f_{i}: A \rightarrow \mathbb{R}_{+}, f_{i}(\bar{y})=\max \left\{p_{i}(\bar{y}-\bar{x}) \mid \bar{x} \in A, \bar{x} \sqsubseteq \bar{y}\right\}$. One can now easily check that the assumptions of the previous lemma are in force. In fact, if $\bar{x} \in B_{i}(\bar{y})$, then there is $\bar{z} \in A$ such that $\bar{z} \sqsubseteq \bar{x} \sqsubset \bar{y}$ and $f_{i}(\bar{x})=p_{i}(\bar{x}-\bar{z})$, so that $f_{i}(\bar{y})-f_{i}(\bar{x}) \geq p_{i}(\bar{y}-\bar{z})-p_{i}(\bar{x}-\bar{z})=p_{i}(\bar{y}-\bar{x}) \geq \varkappa_{n}|\bar{y}-\bar{x}| \geq \sigma(\bar{y})$. Therefore there are $\bar{x}, \bar{y} \in A$ and $i \in n$ such that $\bar{x} \sqsubseteq \bar{y}$ and

$$
\delta(A) \geq|\bar{x}-\bar{y}| \geq p_{i}(\bar{y}-\bar{x})=f_{i}(\bar{y}) \geq \frac{1}{2} \sqrt[n]{\sum_{\bar{z} \in A} \sigma(\bar{z})^{n}} .
$$

3. Ultrametric spaces and bi-Lipschitz embeddings. The results of this section rely heavily on the fact that the open balls of fixed radius $r$ form a partition of an ultrametric space. This has some nice structural consequences, which have been described in [LM-L, Theorem 2.2 and Remarks 2.3]. In the special case when the ultrametric space is bounded, it has treelike structure (cf. Example 3.2 here, where the space is actually like a binary tree). In the sequel, the special properties of ultrametric spaces and the result of the previous section are applied to the bi-Lipschitz embeddability of an ultrametric space in the Euclidean space $\mathbb{R}^{n}$.

3.1. Definition. Let $(X, d)$ and $\left(Y, d^{\prime}\right)$ be metric spaces.

(a) A function $f: X \rightarrow Y$ is a bi-Lipschitz embedding if there is a real number $L \geq 1$ such that for every $x, y \in X$,

$$
\frac{1}{L} d(x, y) \leq d^{\prime}(f(x), f(y)) \leq L d(x, y) .
$$

If there is a need to stress the constant (the bi-Lipschitz coefficient) L, the function $f$ is also called an L-bi-Lipschitz embedding. 1-bi-Lipschitz embeddings are called isometric embeddings. If only the rightmost inequality is required, for some $L \geq 0$ and every $x, y \in X$, then $f$ is called a Lipschitz (or an L-Lipschitz) function.

(b) The space $(X, d)$ is an ultrametric space (and $d$ is an ultrametric) if for every $x, y, z \in X$, the strong triangle inequality $d(x, z) \leq \max \{d(x, y)$, $d(y, z)\}$ holds. 
Let $(X, d)$ be an ultrametric space and $n \in \mathbb{N}^{*}$. I shall define a function $\nu_{n}: \mathcal{P}_{\omega}^{*}(X) \rightarrow \mathbb{R}_{+}$which will be of help in measuring the diameter of the image $f[Y]$ of a finite set $Y \subset X$ under a bi-Lipschitz embedding $f: X \rightarrow$ $\mathbb{R}^{n}$. To this aim, note first that

$$
\sim_{r}=\{(x, y) \in X \times X \mid d(x, y)<r\}
$$

is an equivalence relation on $X$ for every $r \in \mathbb{R}_{+}^{*}$, as $(X, d)$ is ultrametric. Obviously, $\sim_{r} \subset \sim_{s}$ whenever $0<r \leq s$.

Fix $Y \in \mathcal{P}_{\omega}^{*}(X)$ for a while. For $r \in \mathbb{R}_{+}^{*}$, let $k(r)$ be the number of the equivalence classes of $\sim_{r}$ which meet $Y$. Then clearly $k: \mathbb{R}_{+}^{*} \rightarrow \mathbb{N}$ is a left-continuous decreasing function, and the support $\operatorname{supt}(w)=\left\{r \in \mathbb{R}_{+}^{*} \mid\right.$ $w(r) \neq 0\}$ of the function $w: \mathbb{R}_{+}^{*} \rightarrow \mathbb{N}, w(r)=k(r)-\lim _{x \rightarrow r+} k(x)$, is finite. Moreover, $a(Y)=\min \operatorname{supt}(w)$ provided that $|Y| \geq 2$, and $\sum_{r \in \operatorname{supt}(w)} w(r)$ $=|Y|-1$. Now set

$$
\nu_{n}(Y)=\sum_{r>0} w(r) r^{n}=\sum_{r \in \operatorname{supt}(w)} w(r) r^{n} .
$$

There is a very canonical class of ultrametric spaces, namely the ultrametric Cantor spaces. In the course of the proof it is instructive to follow what happens in this particular case.

3.2. Example. Let $C={ }^{\mathbb{N}}\{0,1\}$ be the set of all functions $f: \mathbb{N} \rightarrow\{0,1\}$. There are various ways to endow $C$ with an ultrametric; specifically, for every $\alpha>1$, let

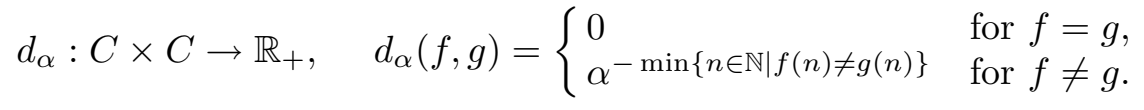

Then $\left(C, d_{\alpha}\right)$ is an ultrametric space. Since $d_{\alpha}$ induces the product topology on $C$, the space $\left(C, d_{\alpha}\right)$ is compact. Let us fix $\alpha$, and consider a finite $Y \subset C$ with at least two points. Obviously, $\delta(Y)=\alpha^{-l}$ and $a(Y)=\alpha^{-m}$ for some $l, m \in \mathbb{N}, l \leq m$, and the restriction $f \nmid\{0, \ldots, l-1\}$ does not depend on $f \in Y$. Let us use the same notation $k, w$ as above. Then it is straightforward to check that $k(r)=k\left(\alpha^{-j}\right)$ for $j \in \mathbb{N}$ and $\left.\left.r \in\right] \alpha^{-j-1}, \alpha^{-j}\right]$, that $\operatorname{supt}(w) \subset$ $\left\{\alpha^{-j} \mid j \in \mathbb{N}, l \leq j \leq m\right\}$, and that for every $j \in \mathbb{N}$, we have $k\left(\alpha^{-l-j}\right) \leq$ $2^{j+1}$ and $w\left(\alpha^{-l-j}\right) \leq 2^{j}$. In particular,

$$
\begin{aligned}
|Y| & =k(a(Y))=k\left(\alpha^{-l-(m-l)}\right) \\
& \leq 2^{m-l+1}=2 \cdot\left(\alpha^{m-l}\right)^{s}=2 \cdot\left(\frac{\delta(Y)}{a(Y)}\right)^{s},
\end{aligned}
$$

where $s=\log _{\alpha} 2=\ln 2 / \ln \alpha$. Later we learn that $s$ is a good dimensional parameter. 
Let $n \in \mathbb{N}$. For the function $\nu_{n}$ we get the estimate

$$
\begin{aligned}
\nu_{n}(Y) & =\sum_{j=0}^{m-l} w\left(\alpha^{-l-j}\right)\left(\alpha^{-l-j}\right)^{n} \\
& \leq \alpha^{-n l} \sum_{j=0}^{m-l} 2^{j} \alpha^{-n j}=\delta(Y)^{n} \sum_{j=0}^{m-l}\left(\frac{2}{\alpha^{n}}\right)^{j} .
\end{aligned}
$$

These inequalities are the best possible in the sense that there are sets $Y$ of arbitrarily large cardinality (and even of arbitrarily small diameter) such that equalities hold in (A) and (B). Indeed, given $f \in C$ and $l, m \in \mathbb{N}$ with $l \leq m$, let $Y$ be the set of all $g \in C$ such that $g\lceil\{0, \ldots, l-1\}=f\lceil\{0, \ldots, l-$ $1\}$ and $g(j)=0$, for every $j \in \mathbb{N}, j>m$. Then $|Y|=2^{m-l+1}, \delta(Y)=\alpha^{-l}$ and $a(Y)=\alpha^{-m}$, which implies $|Y|=2(\delta(Y) / a(Y))^{s}$. Furthermore we have $k\left(\alpha^{-l-j}\right)=2^{j+1}$ and $w\left(\alpha^{-l-j}\right)=2^{j}$, for $j \in \mathbb{N}, j \leq m-l$, so that equality holds in (B), too.

We have the following cases: If $n>s$, then $2 / \alpha^{n}<1$ and the geometric series $\sum_{j=0}^{\infty}\left(2 / \alpha^{n}\right)^{j}$ converges, which gives an upper bound for the ratio $\nu_{n}(Y) / \delta(Y)^{n}$. If $n=s$, we know that for every $l, m \in \mathbb{N}, l \leq m$, there exists $Y$ such that $\nu_{n}(Y)=(m-l+1) \delta(Y)^{n}$. Hence, no upper bound can be given for $\nu_{n}(Y) / \delta(Y)^{n}$. Note that this applies to $\nu_{n}(Y)$ as well, since if we choose $l=0$, we have $\delta(Y)=1$. Similarly, if $n<s$, the fact that $\sum_{j=0}^{\infty}\left(2 / \alpha^{n}\right)^{j}$ diverges implies that there is no upper bound for $\nu_{n}(Y) / \delta(Y)^{n}$.

3.3. Lemma. Let $(X, d)$ be an ultrametric space, $n \in \mathbb{N}^{*}$ and $Y \subset X$ a finite set with at least two points. Denote by $\mathcal{Y}$ the partition of $Y$ corresponding to the equivalence relation $\sim_{\delta(Y)} \cap(Y \times Y)$. Then $|\mathcal{Y}| \geq 2$ and

$$
\nu_{n}(Y)=(|\mathcal{Y}|-1) \delta(Y)^{n}+\sum_{Z \in \mathcal{Y}} \nu_{n}(Z)
$$

Proof. As $\delta(Y)>0$, there are points of $Y$ which are inequivalent with respect to $\sim_{\delta(Y)}$, whence $|\mathcal{Y}| \geq 2$. For every $Z \in \mathcal{Y}$ and $r \in \mathbb{R}_{+}^{*}$, let $k_{Z}(r)$ be the number of the equivalence classes of $\sim_{r}$ which meet $Z$, and let $w_{Z}: \mathbb{R}_{+}^{*} \rightarrow \mathbb{N}, w_{Z}(r)=k_{Z}(r)-\lim _{x \rightarrow r+} k_{Z}(x)$. Moreover, let $k$ and $w$ be the corresponding functions for $Y$. Then one easily checks that $k(r)=\sum_{Z \in \mathcal{Y}} k_{Z}(r)$, for every $r \leq \delta(Y)$, which implies

$$
\begin{aligned}
w(\delta(Y)) & =k(\delta(Y))-1=\left(\sum_{Z \in \mathcal{Y}} k_{Z}(\delta(Y))\right)-1 \\
& =\sum_{Z \in \mathcal{Y}}\left(k_{Z}(\delta(Y))-1\right)+|\mathcal{Y}|-1=|\mathcal{Y}|-1+\sum_{Z \in \mathcal{Y}} w_{Z}(\delta(Y))
\end{aligned}
$$


and for every $r<\delta(Y), w(r)=\sum_{Z \in \mathcal{Y}} w_{Z}(r)$. Hence by definition,

$$
\begin{aligned}
\nu_{n}(Y) & =\sum_{r>0} w(r) r^{n}=\sum_{r \in] 0, \delta(Y)[} w(r) r^{n}+w(\delta(Y)) \delta(Y)^{n} \\
& =\sum_{r \in] 0, \delta(Y)[} \sum_{Z \in \mathcal{Y}} w_{Z}(r) r^{n}+\delta(Y)^{n}\left(|\mathcal{Y}|-1+\sum_{Z \in \mathcal{Y}} w_{Z}(\delta(Y))\right) \\
& =(|\mathcal{Y}|-1) \delta(Y)^{n}+\sum_{Z \in \mathcal{Y}} \sum_{r>0} w_{Z}(r) r^{n} \\
& =(|\mathcal{Y}|-1) \delta(Y)^{n}+\sum_{Z \in \mathcal{Y}} \nu_{n}(Z) .
\end{aligned}
$$

The following proposition gives an alternative definition for the function $\nu_{n}$ in terms of linear orders.

3.4. Proposition. Suppose $(X, d)$ is an ultrametric space and $\preceq$ is a linear order on $X$. Then for every $Y \in \mathcal{P}_{\omega}^{*}(X)$,

$$
\nu_{n}(Y)=\sum_{x \in Y} \sigma_{Y}(x)^{n}
$$

where

$\sigma_{Y}: Y \rightarrow \mathbb{R}_{+}, \quad \sigma_{Y}(x)= \begin{cases}0 & \text { for } x=\min _{\preceq} Y, \\ \min \{d(y, x) \mid y \in Y, y \prec x\} & \text { otherwise. }\end{cases}$

Proof. The proof proceeds by induction on the cardinality of $Y$. The claim is clearly true if $Y=\{y\}$ is a singleton, as then $\nu_{n}(Y)=0=\sigma_{Y}(y)$, so let us assume that $|Y| \geq 2$. Let $\mathcal{Y}$ be the partition defined in the previous lemma. For $Z \in \mathcal{Y}$ and $z \in Z$, let us compare the sets of predecessors $P=\{x \in Y \mid x \prec z\}$ and $Q=\{x \in Z \mid x \prec z\}$. Since $Z$ is a $\sim_{\delta(Y)} \cap(Y \times Y)$ equivalence class, $P \backslash Q=\{x \in Y \mid x \prec z, d(x, z)=\delta(Y)\}$. This readily gives the comparison of the values $\sigma_{Y}(z)$ and $\sigma_{Z}(z)$ for the occurring three cases: If $z=\min _{\preceq}$, then also $z=\min _{\preceq} Z$ and $\sigma_{Y}(z)=\sigma_{Z}(z)=0$. If $z=\min _{\preceq} Z \neq \min _{\preceq} Y$, then $P \backslash Q \neq \emptyset$, so that $\sigma_{Y}(z)=\delta(Y)$ and $\sigma_{Z}(z)=0$. Finally, if $z \neq \min _{\preceq} Z$, then

$$
\begin{aligned}
\sigma_{Y}(z) & =\min \{d(x, z) \mid x \in P\}=\min (\{\delta(Y)\} \cup\{d(x, z) \mid x \in Q\}) \\
& =\min \{d(x, z) \mid x \in Q\}=\sigma_{Z}(z),
\end{aligned}
$$

because $d(x, z)<\delta(Y)$, for every $x \in Q$. Altogether, using the notation $x_{Z}=\min _{\preceq} Z$ and applying the induction hypothesis, for each $Z \in \mathcal{Y}$, we have

$$
\sum_{x \in Y} \sigma_{Y}(x)^{n}=\sum_{Z \in \mathcal{Y}}\left(\sigma_{Y}\left(x_{Z}\right)^{n}+\sum_{x \in Z \backslash\left\{x_{Z}\right\}} \sigma_{Y}(x)^{n}\right)
$$




$$
\begin{aligned}
& =(|\mathcal{Y}|-1) \delta(Y)^{n}+\sum_{Z \in \mathcal{Y}} \sum_{x \in Z} \sigma_{Z}(x)^{n} \\
& =(|\mathcal{Y}|-1) \delta(Y)^{n}+\sum_{Z \in \mathcal{Y}} \nu_{n}(Z)=\nu_{n}(Y),
\end{aligned}
$$

by the previous lemma, and the induction is completed.

Observe that, in the previous proposition, even the set $\sigma_{Y}[Y]=\left\{\sigma_{Y}(x) \mid\right.$ $x \in Y\}$ is independent of the linear order $\preceq$. This is because $\sigma_{Y}[Y]$ obeys the following recursion formula, which can be deduced along the same lines as the proof itself: $\sigma_{Y}[Y]=\{0\}$, for a singleton $Y \in \mathcal{P}_{\omega}^{*}(X)$, and $\sigma_{Y}[Y]=$ $\bigcup_{Z \in \mathcal{Y}} \sigma_{Z}[Z] \cup\{\delta(Y)\}$, where $\mathcal{Y}$ is as defined in Lemma 3.3, for $Y \in \mathcal{P}_{\omega}^{*}(X)$, $|Y| \geq 2$.

The next step is to show that $\nu_{n}(Y)$ is related to the size of the image $f[Y]$.

3.5. Lemma. Suppose $f$ is an injection of an ultrametric space $(X, d)$ in the Euclidean space $\mathbb{R}^{n}$ such that $f^{-1}$ is L-Lipschitz with $L>0$. Then for every $Y \in \mathcal{P}_{\omega}^{*}(X)$,

$$
\delta(f[Y]) \geq \frac{\varkappa_{n}}{2 L} \sqrt[n]{\nu_{n}(Y)} .
$$

Proof. The linear order $\sqsubseteq$ and the injection $f$ induce a natural linear order $\preceq$ on $X$, namely $\preceq=\{(x, y) \in X \times X \mid f(x) \sqsubseteq f(y)\}$. Let $Y \in \mathcal{P}_{\omega}^{*}(X)$ be arbitrary and let $\sigma, \sigma_{Y}$ be as in Propositions 2.2 and 3.4 with $A=f[Y]$. Since $f^{-1}$ is an $L$-Lipschitz function, $\sigma(f(x)) \geq \varkappa_{n} \sigma_{Y}(x) / L$, for every $x \in Y \backslash\left\{\min _{\preceq}\right\}$. Using Propositions 2.2 and 3.4 (and their notation) we get

$$
\begin{aligned}
\delta(f[Y]) & \geq \frac{1}{2} \sqrt[n]{\sum_{x \in Y} \sigma(f(x))^{n}} \geq \frac{1}{2} \sqrt[n]{\sum_{x \in Y}\left(\frac{\varkappa_{n}}{L} \sigma_{Y}(x)\right)^{n}} \\
& =\frac{\varkappa_{n}}{2 L} \sqrt[n]{\sum_{x \in Y} \sigma_{Y}(x)^{n}}=\frac{\varkappa_{n}}{2 L} \sqrt[n]{\nu_{n}(Y)}
\end{aligned}
$$

3.6. Example. Consider the compact ultrametric space $\left(C, d_{\alpha}\right)$ in the special case $\alpha=2^{1 / n}$ with $n \in \mathbb{N}^{*}$. Let us show that there is no bounded injection, and in particular, no topological embedding, $f: C \rightarrow \mathbb{R}^{n}$ for which $f^{-1}: f[C] \rightarrow C$ is a Lipschitz function. Suppose on the contrary that such a function $f$ exists. Choose $L \geq 1$ such that $f^{-1}$ is $L$-Lipschitz; then for every $Y \in \mathcal{P}_{\omega}^{*}(C)$, we have $\delta(f[C]) \geq \delta(f[Y]) \geq\left(\varkappa_{n} /(2 L)\right) \sqrt[n]{\nu_{n}(Y)}$, by the previous lemma. But on the other hand, by Example 3.2, $\nu_{n}(Y)$ can be arbitrarily large, which is in contradiction with the hypothesis that $f$ is bounded. 


\section{Bi-Lipschitz embeddability and Assouad dimension}

4.1. Definition. For $C, s \in \mathbb{R}_{+}$, a metric space $(X, d)$ is $(C, s)$-homogeneous if the inequality

$$
\left|X_{0}\right| \leq C\left(\frac{b}{a}\right)^{s}
$$

holds for $a, b \in \mathbb{R}_{+}^{*}$ and $X_{0} \subset X$ provided that $b \geq a$ and that for every distinct $x, y \in X_{0}, a \leq d(x, y) \leq b$ holds. The space $(X, d)$ is $s$-homogeneous if it is $(C, s)$-homogeneous for some $C \in \mathbb{R}_{+}$. The Assouad dimension of $(X, d)$ is

$$
\operatorname{dim}_{\mathrm{A}}(X, d)=\inf \left\{s \in \mathbb{R}_{+} \mid(X, d) \text { is } s \text {-homogeneous }\right\},
$$

if this infimum exists; otherwise $\operatorname{dim}_{\mathrm{A}}(X, d)=\infty$.

The following expected result (due to Assouad [A]) is a good illustration of the dimensional contents of Proposition 2.2.

4.2. Proposition. Let $n \in \mathbb{N}^{*}$ and equip $\mathbb{R}^{n}$ with the usual Euclidean metric d. Then $\operatorname{dim}_{\mathrm{A}}\left(\mathbb{R}^{n}, d\right)=n$.

Proof. Let $s<n$ and consider $A_{l}=\{0, \ldots, l\}^{n}$, for $l \in \mathbb{N}^{*}$. Then

$$
\lim _{l \rightarrow \infty}\left|A_{l}\right| /\left(\frac{\delta\left(A_{l}\right)}{a\left(A_{l}\right)}\right)^{s}=\lim _{l \rightarrow \infty} \frac{(l+1)^{n}}{(l \sqrt{n})^{s}}=\infty,
$$

so that $\left(\mathbb{R}^{n}, d\right)$ is not $s$-homogeneous. On the other hand, suppose $A \subset \mathbb{R}^{n}$ is a finite set with at least two points. Let $\sigma$ be as in Proposition 2.2; then $\sigma(\bar{x}) \geq \varkappa_{n} a(A)$, for every $\bar{x} \in A \backslash\left\{\min _{\sqsubseteq} A\right\}$. By Proposition 2.2,

$$
\begin{aligned}
\delta(A) & \geq \frac{1}{2} \sqrt[n]{\sum_{\bar{x} \in A} \sigma(\bar{x})^{n}} \geq \frac{1}{2} \sqrt[n]{(|A|-1)\left(\varkappa_{n} a(A)\right)^{n}} \\
& \geq \frac{1}{2} \varkappa_{n} a(A) \sqrt[n]{\frac{|A|}{2}} \geq \frac{\varkappa_{n} a(A)}{4} \sqrt[n]{|A|},
\end{aligned}
$$

which implies that

$$
|A| \leq\left(\frac{4 \delta(A)}{\varkappa_{n} a(A)}\right)^{n} .
$$

Hence, $\left(\mathbb{R}^{n}, d\right)$ is $\left(\left(4 / \varkappa_{n}\right)^{n}, n\right)$-homogeneus and $\operatorname{dim}_{\mathrm{A}}\left(\mathbb{R}^{n}, d\right)=n$.

Another example is the Cantor space $\left(C, d_{\alpha}\right), \alpha>1$, from Example 3.2. By inequality (A) there, $\left(C, d_{\alpha}\right)$ is $(2, s)$-homogeneous for $s=\log _{\alpha} 2=$ $\ln 2 / \ln \alpha$. It was also mentioned in the example that inequality can be replaced by equality for finite subsets $Y \subset C$ of arbitrarily large cardinality. Hence, $\operatorname{dim}_{\mathrm{A}}\left(C, d_{\alpha}\right)=s$. Compare also Example 3.6, where $s=n$. 
At this last stage it will be shown that it is possible to find an upper bound for $\operatorname{dim}_{\mathrm{A}}(X, d)$ from the bi-Lipschitz embedding properties of the space $(X, d)$. For that purpose, a technical lemma is necessary.

4.3. Lemma. Let $c \in] 0,1\left[\right.$ and $\left.k=\left(1-\log _{2}(1-c)\right)^{-1} \in\right] 0,1[$. Then $f:] 0,1 / c\left[\rightarrow \mathbb{R}, f(x)=(1-c x)^{k}(1+x)^{1-k}\right.$, is a function such that $f(x) \leq 1$, for every $x \in[1,1 / c[$.

Proof. The function $f$ is differentiable and $f^{\prime}(x)=f(x) h(x)$, for every $x \in] 0,1 / c[$, where $h:] 0,1 / c[\rightarrow \mathbb{R}, h(x)=-k c /(1-c x)+(1-k) /(1+x)$. The constant $k$ is so chosen that $f(1)=(1-c)^{k} \cdot 2^{1-k}=2^{k \log _{2}(1-c)+1-k}=2^{0}=1$. Therefore, it is enough to show that $f^{\prime}(x)<0$, for every $\left.x \in\right] 1,1 / c[$.

Consider the value

$$
\begin{aligned}
h(1) & =f^{\prime}(1) / f(1)=\frac{-k c}{1-c}+\frac{1-k}{2} \\
& =\frac{c+1+(c-1) k^{-1}}{2 k^{-1}(c-1)}=\frac{2 c+(1-c) \log _{2}(1-c)}{2 k^{-1}(c-1)} .
\end{aligned}
$$

The denominator is obviously negative. To estimate the numerator, let $g$ : ]$-\infty, 1\left[\rightarrow \mathbb{R}, g(x)=2 x+(1-x) \log _{2}(1-x)=2 x+(1-x) \ln (1-x) / \ln 2\right.$. Then $g(0)=0, g$ is differentiable and $g^{\prime}(x)=2-(1+\ln (1-x)) / \ln 2$, for every $x<1$. Since $g^{\prime}(0)=2-1 / \ln 2>0$ and $g^{\prime}$ is increasing, $g^{\prime}(x)>0$ holds, for every $x \in] 0,1[$. Hence, $g(c)>0$, which implies $h(1)<0$.

The function $f$ takes only positive values; in particular, $f^{\prime}(1)=h(1) f(1)$ $<0$. One readily sees that $h$ is decreasing on $[1,1 / c[$, whence for every $x \in\left[1,1 / c\left[, h(x) \leq h(1)<0\right.\right.$, and consequently $f^{\prime}(x)<0$.

4.4. Proposition. Let $(X, d)$ be an ultrametric space L-bi-Lipschitz embeddable in $\mathbb{R}^{n}$. Suppose $Y \subset X$ is a finite set with at least two points. Then $\delta(Y)^{n} \geq C_{0} \nu_{n}(Y)$ and $|Y| \leq C_{1}\left(\nu_{n}(Y) / a(Y)^{n}\right)^{k}$, where $C_{0}=\left(\varkappa_{n} /\left(2 L^{2}\right)\right)^{n}$ $\in] 0,1\left[, C_{1}=\left(1-\left(1-C_{0}\right)^{k}\right)^{-1}>1\right.$ and $\left.k=\left(1-\log _{2}\left(1-C_{0}\right)\right)^{-1} \in\right] 0,1[$.

Proof. By assumption, there is an $L$-bi-Lipschitz embedding $f: X \rightarrow$ $\mathbb{R}^{n}$. Lemma 3.5 implies that for every $Y \in \mathcal{P}_{\omega}^{*}(X)$, we have $\delta(Y) \geq$ $\delta(f[Y]) / L \geq\left(\varkappa_{n} /\left(2 L^{2}\right)\right) \sqrt[n]{\nu_{n}(Y)}=\sqrt[n]{C_{0} \nu_{n}(Y)}$. It is easy to check that $\left.\left.C_{0} \in\right] 0,1 / 2\right], C_{1} \geq 2$ and $\left.k \in\right] 0,1[$. The claim of the proposition is proved by induction on the cardinality of $Y$. Observe that by the definition of $\nu_{n}$ and since $|Y| \geq 2$, the inequality $\nu_{n}(Y) \geq a(Y)^{n}>0$ holds. As $C_{1} \geq 2$, the starting case $|Y|=2$ is part of Step 1.

1) Assume $|Y| \leq C_{1}$. Then $C_{1}\left(\nu_{n}(Y) / a(Y)^{n}\right)^{k} \geq C_{1} \geq|Y|$.

2) Assume $|Y|>C_{1} \geq 2$. Let $\mathcal{Y}$ be the partition from Lemma 3.3. Then $|\mathcal{Y}| \geq 2$ and $\nu_{n}(Y)=(|\mathcal{Y}|-1) \delta(Y)^{n}+\sum_{Z \in \mathcal{Y}} \nu_{n}(Z)$. Here we have two subcases according to whether $\mathcal{Y}$ contains singletons or not. 
2a) Suppose $\left\{x_{0}\right\} \in \mathcal{Y}$ for some $x_{0} \in Y$. Let $Y_{0}=Y \backslash\left\{x_{0}\right\}$; if $\mathcal{Y}=$ $\left\{Y_{0},\left\{x_{0}\right\}\right\}$, then $\nu_{n}(Y)=(2-1) \delta(Y)^{n}+\nu_{n}\left(Y_{0}\right)+\nu_{n}\left(\left\{x_{0}\right\}\right)=1 \cdot \delta(Y)^{n}+$ $\nu_{n}\left(Y_{0}\right)+0=\nu_{n}\left(Y_{0}\right)+\delta(Y)^{n}$. On the other hand, if $Y_{0} \notin \mathcal{Y}$, then there are $y_{1}, y_{2} \in Y_{0}$ such that $y_{1} \chi_{\delta(Y)} y_{2}$, because $\mathcal{Y}$ is the partition corresponding to the equivalence relation $\sim_{\delta(Y)} \cap(Y \times Y)$. Hence $\delta\left(Y_{0}\right)=\delta(Y)$ and the partition of $Y_{0}$ related to $\sim_{\delta\left(Y_{0}\right)} \cap\left(Y_{0} \times Y_{0}\right)$ is simply $\mathcal{Y}_{0}=\mathcal{Y} \backslash\left\{\left\{x_{0}\right\}\right\}$. Applying Lemma 3.3 to both $Y$ and $Y_{0}$ in place of $Y$ (note that $\left|Y_{0}\right| \geq 2$ ) we get $\nu_{n}(Y)-\nu_{n}\left(Y_{0}\right)=\left(|\mathcal{Y}|-\left|\mathcal{Y}_{0}\right|\right) \delta(Y)^{n}+\sum_{Z \in \mathcal{Y} \backslash \mathcal{Y}_{0}} \nu_{n}(Z)=1 \cdot \delta(Y)^{n}+$ $\nu_{n}\left(\left\{x_{0}\right\}\right)=\delta(Y)^{n}$, so that $\nu_{n}(Y)=\nu_{n}\left(Y_{0}\right)+\delta(Y)^{n}$ holds anyway.

By the induction hypothesis, $\left|Y_{0}\right| \leq C_{1}\left(\nu_{n}\left(Y_{0}\right) / a\left(Y_{0}\right)^{n}\right)^{k}$. Moreover, $\delta(Y)$ $\geq \sqrt[n]{C_{0} \nu_{n}(Y)}$ implies

$$
\frac{\nu_{n}(Y)}{\nu_{n}\left(Y_{0}\right)}=\frac{\nu_{n}(Y)}{\nu_{n}(Y)-\delta(Y)^{n}} \geq \frac{\nu_{n}(Y)}{\nu_{n}(Y)-C_{0} \nu_{n}(Y)}=\frac{1}{1-C_{0}} .
$$

Consequently,

$$
\begin{aligned}
|Y| & =\left|Y_{0}\right| \frac{|Y|}{|Y|-1} \leq\left|Y_{0}\right| \frac{C_{1}}{C_{1}-1} \leq C_{1}\left(\frac{\nu_{n}\left(Y_{0}\right)}{a\left(Y_{0}\right)^{n}}\right)^{k}\left(1-C_{0}\right)^{-k} \\
& \leq C_{1}\left(\frac{\nu_{n}\left(Y_{0}\right)}{a(Y)^{n}\left(1-C_{0}\right)}\right)^{k} \leq C_{1}\left(\frac{\nu_{n}(Y)}{a(Y)^{n}}\right)^{k} .
\end{aligned}
$$

2b) Suppose $|Z| \geq 2$ for every $Z \in \mathcal{Y}$. Since $k \in] 0,1[$, the function $x \mapsto x^{k}$ is concave and owing to the induction hypothesis and a well-known inequality concerning concave functions we get

$$
\begin{aligned}
|Y| & =\sum_{Z \in \mathcal{Y}}|Z| \leq \sum_{Z \in \mathcal{Y}} C_{1}\left(\frac{\nu_{n}(Z)}{a(Z)^{n}}\right)^{k} \leq \sum_{Z \in \mathcal{Y}} C_{1}\left(\frac{\nu_{n}(Z)}{a(Y)^{n}}\right)^{k} \\
& =\frac{C_{1}|\mathcal{Y}|}{a(Y)^{n k}} \sum_{Z \in \mathcal{Y}} \frac{\nu_{n}(Z)^{k}}{|\mathcal{Y}|} \leq \frac{C_{1}|\mathcal{Y}|}{a(Y)^{n k}}\left(\frac{\sum_{Z \in \mathcal{Y}} \nu_{n}(Z)}{|\mathcal{Y}|}\right)^{k} \\
& =\frac{C_{1}|\mathcal{Y}|^{1-k}}{a(Y)^{n k}}\left(\sum_{Z \in \mathcal{Y}} \nu_{n}(Z)\right)^{k} .
\end{aligned}
$$

On the other hand,

$$
\begin{aligned}
\nu_{n}(Y) & =(|\mathcal{Y}|-1) \delta(Y)^{n}+\sum_{Z \in \mathcal{Y}} \nu_{n}(Z) \\
& \geq C_{0}(|\mathcal{Y}|-1) \nu_{n}(Y)+\sum_{Z \in \mathcal{Y}} \nu_{n}(Z)
\end{aligned}
$$

and thus $\left(1-C_{0}(|\mathcal{Y}|-1)\right) \nu_{n}(Y) \geq \sum_{Z \in \mathcal{Y}} \nu_{n}(Z)$. Applying Lemma 4.3 with $c=C_{0}$ and $x=|\mathcal{Y}|-1 \geq 1$, which is possible as $1-c x>0$, gives 


$$
\begin{aligned}
|Y| & \leq \frac{C_{1}|\mathcal{Y}|^{1-k}}{a(Y)^{n k}}\left(\sum_{Z \in \mathcal{Y}} \nu_{n}(Z)\right)^{k} \\
& \leq \frac{C_{1} \nu_{n}(Y)^{k}}{a(Y)^{n k}}\left(1-C_{0}(|\mathcal{Y}|-1)\right)^{k}|\mathcal{Y}|^{1-k} \leq C_{1}\left(\frac{\nu_{n}(Y)}{a(Y)^{n}}\right)^{k}
\end{aligned}
$$

4.5. Theorem. For every $L \geq 1$ and $n \in \mathbb{N}^{*}$ there are $C \geq 1$ and $s<n$ such that the following holds: Suppose $(X, d)$ is an ultrametric space and there is an L-bi-Lipschitz embedding $f: X \rightarrow \mathbb{R}^{n}$. Then $(X, d)$ is $(C, s)$ homogeneous and thus $\operatorname{dim}_{\mathrm{A}}(X, d)<n$.

Proof. Choose $C=\left(C_{0}{ }^{k}\left(1-\left(1-C_{0}\right)^{k}\right)\right)^{-1}>1$ and $s=n k$, where $C_{0}$ and $k$ are as in the preceding proposition. Then

$$
\begin{aligned}
|Y| & \leq \frac{1}{1-\left(1-C_{0}\right)^{k}}\left(\frac{\nu_{n}(Y)}{a(Y)^{n}}\right)^{k} \\
& \leq \frac{1}{1-\left(1-C_{0}\right)^{k}}\left(\frac{\delta(Y)^{n} / C_{0}}{a(Y)^{n}}\right)^{k}=C\left(\frac{\delta(Y)}{a(Y)}\right)^{s},
\end{aligned}
$$

for every $Y \in \mathcal{P}_{\omega}^{*}(X)$ with at least two elements. If now $Y \subset X$ and $a, b \in$ $\mathbb{R}_{+}^{*}, b \geq a$, are such that $a \leq d(x, y) \leq b$ for every distinct $x, y \in Y$, then either $|Y|<2$ or $a \leq a(Y) \leq \delta(Y) \leq b$. Hence, $|Y| \leq C(b / a)^{s}$.

When this is combined with [LM-L, Theorem 3.8], we get the following corollary.

4.6. Corollary. Let $n$ be a positive integer. Then an ultrametric space $(X, d)$ can be bi-Lipschitz embedded in $\mathbb{R}^{n}$ if and only if $\operatorname{dim}_{\mathrm{A}}(X, d)<n$.

Other known notions of dimension do not seem to be good for characterizing those ultrametric spaces which can be bi-Lipschitz embedded in $\mathbb{R}^{n}$ for fixed $n$, even if we restrict our attention to compact spaces. This is trivial for the topological dimension, since every ultrametric space has a clopen basis and is therefore zero-dimensional. We study the difference of Assouad dimension and Hausdorff dimension $\operatorname{dim}_{\mathrm{H}}$ in some detail.

4.7. ExAmple. Fix $s>0$ and let $\alpha=2^{1 / s}$. Then in the case of Example 3.2 the Assouad and Hausdorff dimensions coincide: $\operatorname{dim}_{\mathrm{H}}\left(C, d_{\alpha}\right)=$ $\operatorname{dim}_{\mathrm{A}}\left(C, d_{\alpha}\right)=s$. On the other hand, it is not too difficult to find a countable compact ultrametric space $\left(Z, d^{\prime \prime}\right)$ with infinite Assouad dimension. Without loss of generality, $C \cap Z=\emptyset$ and the diameter of $Z$ is 1 . Let $Y=C \cup Z$ and $d^{\prime}: Y \times Y \rightarrow \mathbb{R}_{+}$,

$$
d^{\prime}(x, y)= \begin{cases}d_{\alpha}(x, y) & \text { if } x, y \in C \\ d^{\prime \prime}(x, y) & \text { if } x, y \in Z \\ 1 & \text { otherwise }\end{cases}
$$


Then $\left(Y, d^{\prime}\right)$ is a compact ultrametric space for which

$$
\begin{aligned}
\operatorname{dim}_{\mathrm{H}}\left(Y, d^{\prime}\right)=\max & \left\{\operatorname{dim}_{\mathrm{H}}\left(C, d_{\alpha}\right), \operatorname{dim}_{\mathrm{H}}\left(Z, d^{\prime \prime}\right)\right\}=\max \{s, 0\}=s \\
<\infty & =\max \{s, \infty\} \\
& =\max \left\{\operatorname{dim}_{\mathrm{A}}\left(C, d_{\alpha}\right), \operatorname{dim}_{\mathrm{A}}\left(Z, d^{\prime \prime}\right)\right\}=\operatorname{dim}_{\mathrm{A}}\left(Y, d^{\prime}\right) .
\end{aligned}
$$

Consequently, for every $s>0$, there are compact ultrametric spaces $(X, d)$ and $\left(Y, d^{\prime}\right)$ such that $s=\operatorname{dim}_{\mathrm{H}}(X, d)=\operatorname{dim}_{\mathrm{A}}(X, d)$ and $s=\operatorname{dim}_{\mathrm{H}}\left(Y, d^{\prime}\right)<$ $\infty=\operatorname{dim}_{\mathrm{A}}\left(Y, d^{\prime}\right)$, so that any attempts to characterize bi-Lipschitz embeddability of ultrametric spaces in $\mathbb{R}^{n}$ in terms of Hausdorff dimension are bound to fail. By [LM-L, Example 3.6], a similar statement holds for the upper fractal dimension.

5. Approximating isometry. Suppose $(X, d)$ is an ultrametric space and $f: X \rightarrow \mathbb{R}^{n}$ an $L$-bi-Lipschitz embedding. In the preceding section we learnt that not only do we have $\operatorname{dim}_{\mathrm{A}}(X, d)<n$, but also there is an upper bound $s$ for $\operatorname{dim}_{\mathrm{A}}(X, d)$ depending only on $L$ and $n$. A closer look at the proofs of Theorem 4.5 and Proposition 4.4 reveals, however, that the bound there is very modest. Indeed, we chose $s=n k$, where $k=\left(1-\log _{2}\left(1-C_{0}\right)\right)^{-1}$ and $C_{0}=\left(\varkappa_{n} /\left(2 L^{2}\right)\right)^{n}$. For $n=1$, we have $C_{0}=\left(\varkappa_{1} /\left(2 L^{2}\right)\right)^{1}=1 /\left(2 L^{2}\right) \leq$ $1 / 2$ and $k \geq\left(1-\log _{2}(1-1 / 2)\right)^{-1}=1 / 2=1-1 / 2$; if $n \geq 2$, then $C_{0}=$ $\left(\varkappa_{n} /\left(2 L^{2}\right)\right)^{n} \leq\left(\varkappa_{n} / 2\right)^{2}=(4 n(n-1))^{-1} \leq 1 /(4 n)$ and $k \geq 1+\log _{2}\left(1-C_{0}\right) \geq$ $1-2 C_{0} \geq 1-1 /(2 n)$. Hence, $s=n k \geq n(1-1 /(2 n))=n-1 / 2$.

In this section, these bounds will be improved significantly in the special case when $L$ is close to 1 . To be precise, for $L>1$ and $n \in \mathbb{N}^{*}$, let $s(L, n)$ be the supremum over all $\operatorname{dim}_{\mathrm{A}}(X, d)$, where $(X, d)$ is an ultrametric space $L$-bi-Lipschitz embeddable in $\mathbb{R}^{n}$. We shall find out that for every $n \in \mathbb{N}^{*}$, we have $\lim _{L \rightarrow 1+} s(L, n)=0$. Intuitively, if an ultrametric space $(X, d)$ can be embedded in $\mathbb{R}^{n}$ by a bi-Lipschitz function which approximates isometry, then the Assouad dimension of $(X, d)$ is small.

The theorem of this section is simply an elaboration of the fact that only finite ultrametric spaces can be isometrically embedded in Euclidean spaces (see, e.g., [ABBW]). While one reads through the following lemmas, it is instructive to think what happens if the bi-Lipschitz coefficient $L$ is replaced by 1 . Thus, it is easy to see that an ultrametric subspace of $\mathbb{R}$ has at most two points. If $X \subset \mathbb{R}^{n}$ is a finite ultrametric subset with at least two points and $n>1$, it is also relatively simple to find two points $a, b$ of $X$ such that $X \backslash\{a, b\}$ is a subset of some hyperplane of $\mathbb{R}^{n}$. Bearing these two ideas in mind makes it easy to follow the next lemmas.

5.1. Lemma. Let $(X, d)$ be a finite ultrametric space with at least three points. Suppose $(X, d)$ can be L-bi-Lipschitz embedded in $\mathbb{R}$. Then $L \geq$ $\sqrt{1+a(X) / \delta(X)}$. 
Proof. Choose an $L$-bi-Lipschitz embedding $f: X \rightarrow \mathbb{R}$. Since $(X, d)$ is an ultrametric space, we may choose three points $x_{0}, x_{1}, y \in X$ such that $d\left(x_{0}, x_{1}\right)=a(X)$ and $d\left(x_{0}, y\right)=d\left(x_{1}, y\right)=\delta(X)$. If $L \geq \sqrt{2}$, we have nothing to prove, as $\sqrt{1+a(X) / \delta(X)} \leq \sqrt{1+1}=\sqrt{2}$, so assume $1 \leq L<\sqrt{2}$. Then $f(y)$ is not between $f\left(x_{0}\right)$ and $f\left(x_{1}\right)$, since otherwise for $i=0$ or $i=1$ we would have

$$
\begin{aligned}
\frac{\delta(X)}{L} & =\frac{d\left(x_{i}, y\right)}{L} \leq\left|f\left(x_{i}\right)-f(y)\right| \\
& \leq \frac{\left|f\left(x_{0}\right)-f\left(x_{1}\right)\right|}{2} \leq \frac{L}{2} d\left(x_{0}, x_{1}\right)=\frac{L}{2} a(X),
\end{aligned}
$$

implying $\delta(X) / a(X) \leq L^{2} / 2<1$, which is impossible. Therefore, without loss of generality, we may assume that $f\left(x_{1}\right)$ is between $f\left(x_{0}\right)$ and $f(y)$. Then we have

$$
\begin{aligned}
a(X) / L & \leq\left|f\left(x_{0}\right)-f\left(x_{1}\right)\right| \\
& =\left|f\left(x_{0}\right)-f(y)\right|-\left|f\left(x_{1}\right)-f(y)\right| \leq(L-1 / L) \delta(X),
\end{aligned}
$$

which implies $L^{2}-1 \geq a(X) / \delta(X)$, so that $L \geq \sqrt{1+a(X) / \delta(X)}$.

5.2. Lemma. Let $(X, d)$ be a finite ultrametric space with $|X| \geq 2$ and let $f: X \rightarrow \mathbb{R}^{n+1}$ be an L-bi-Lipschitz embedding, where $n \in \mathbb{N}^{*}$. Assume $k=\left(L^{4}-1\right)(\delta(X) / a(X))^{2}<1$ and let $L^{*}=L\left(1-k^{2}\right)^{-1 / 4}$. Then there are points $x_{0}, x_{1} \in X$ and an $L^{*}$-bi-Lipschitz embedding $h: X \backslash\left\{x_{0}, x_{1}\right\} \rightarrow \mathbb{R}^{n}$.

Proof. Choose $x_{0}, x_{1} \in X$ such that $d\left(x_{0}, x_{1}\right)=a(X)$ and denote $X \backslash$ $\left\{x_{0}, x_{1}\right\}$ by $X^{*}$. Let $\bar{e}=\left(f\left(x_{1}\right)-f\left(x_{0}\right)\right) /\left|f\left(x_{1}\right)-f\left(x_{0}\right)\right|$, let $T$ be the set of those $\bar{x} \in \mathbb{R}^{n+1}$ which are perpendicular to $\bar{e}$ and let $c \geq 1$ be a constant to be fixed later. For $y \in X^{*}$, we have the unique representation

$$
f(y)=\frac{f\left(x_{0}\right)+f\left(x_{1}\right)}{2}+\frac{g(y)}{c}+\gamma(y) \bar{e},
$$

where $\gamma: X^{*} \rightarrow \mathbb{R}, \gamma(y)=\left(f(y)-\left(f\left(x_{0}\right)+f\left(x_{1}\right)\right) / 2\right) \cdot \bar{e}$, and $g: X^{*} \rightarrow$ $T, g(y)=c\left(f(y)-\left(f\left(x_{0}\right)+f\left(x_{1}\right)\right) / 2-\gamma(y) \bar{e}\right)$. We intend to show that $f(y)-\left(f\left(x_{0}\right)+f\left(x_{1}\right)\right) / 2$ is close to the hyperplane $T$, or in other words, $|\gamma(y)|$ is small. (If $L=1$, we simply have $\gamma(y)=0$.)

Let $u=\left|f\left(x_{1}\right)-f\left(x_{0}\right)\right|$. For $y \in X^{*}$ and $i \in\{0,1\}$, we have

$$
\left|f(y)-f\left(x_{i}\right)\right|^{2}=|g(y)|^{2} / c^{2}+\left(\gamma(y)+(-1)^{i} u / 2\right)^{2},
$$

so that

$\left|f(y)-f\left(x_{0}\right)\right|^{2}-\left|f(y)-f\left(x_{1}\right)\right|^{2}=(\gamma(y)+u / 2)^{2}-(\gamma(y)-u / 2)^{2}=2 \gamma(y) u$.

On the other hand, as $(X, d)$ is an ultrametric space and $d\left(y, x_{i}\right) \geq a(X)=$ $d\left(x_{0}, x_{1}\right)$, for $i=0,1$, we know that $d\left(y, x_{0}\right)=d\left(y, x_{1}\right)=b$, where $b$ depends on $y$, of course. Since $f$ is an $L$-bi-Lipschitz embedding, we have

$$
\left(1 / L^{2}-L^{2}\right) b^{2} \leq\left|f(y)-f\left(x_{0}\right)\right|^{2}-\left|f(y)-f\left(x_{1}\right)\right|^{2} \leq\left(L^{2}-1 / L^{2}\right) b^{2},
$$


so that

$$
\begin{aligned}
\frac{|\gamma(y)|}{a(X)} & =\frac{|| f(y)-\left.f\left(x_{0}\right)\right|^{2}-\left|f(y)-f\left(x_{1}\right)\right|^{2} \mid}{2 u a(X)} \leq \frac{\left(L^{2}-1 / L^{2}\right) b^{2}}{2 u a(X)} \\
& \leq \frac{\left(L^{2}-1 / L^{2}\right) \delta(X)^{2}}{2 a(X)^{2} / L}=\frac{L^{4}-1}{2 L}\left(\frac{\delta(X)}{a(X)}\right)^{2}=\frac{k}{2 L} .
\end{aligned}
$$

Now that we know that $|\gamma(y)|$ is small, let us proceed to show that with the right choice of $c$, the function $g$ is an $L^{*}$-bi-Lipschitz embedding. For every $y, y^{\prime} \in X^{*}, y \neq y^{\prime}$, we have

$$
\left|f(y)-f\left(y^{\prime}\right)\right|^{2}=\frac{1}{c^{2}}\left|g(y)-g\left(y^{\prime}\right)\right|^{2}+\left(\gamma(y)-\gamma\left(y^{\prime}\right)\right)^{2},
$$

implying that

$$
\begin{aligned}
\frac{1}{c} \cdot \frac{\left|g(y)-g\left(y^{\prime}\right)\right|}{\left|f(y)-f\left(y^{\prime}\right)\right|} & =\sqrt{1-\frac{\left(\gamma(y)-\gamma\left(y^{\prime}\right)\right)^{2}}{\left|f(y)-f\left(y^{\prime}\right)\right|^{2}}} \\
& \geq \sqrt{1-\frac{\left(\gamma(y)-\gamma\left(y^{\prime}\right)\right)^{2}}{(a(X) / L)^{2}}} \geq \sqrt{1-\left(L \frac{|\gamma(y)|+\left|\gamma\left(y^{\prime}\right)\right|}{a(X)}\right)^{2}} \\
& \geq \sqrt{1-\left(L \cdot 2 \cdot \frac{k}{2 L}\right)^{2}}=\sqrt{1-k^{2}} .
\end{aligned}
$$

But trivially $\left|g(y)-g\left(y^{\prime}\right)\right| \leq c\left|f(y)-f\left(y^{\prime}\right)\right|$, so that if we fix $c$ to be $\left(1-k^{2}\right)^{-1 / 4} \geq 1$, we get

$$
\frac{1}{c L} d\left(y, y^{\prime}\right) \leq c \sqrt{1-k^{2}} \cdot\left|f(y)-f\left(y^{\prime}\right)\right| \leq\left|g(y)-g\left(y^{\prime}\right)\right| \leq c L d\left(y, y^{\prime}\right),
$$

as $c^{-2}=\sqrt{1-k^{2}}$. Because $L^{*}=L\left(1-k^{2}\right)^{-1 / 4}=c L$ and there is a natural isometry $j: T \rightarrow \mathbb{R}^{n}$, the composition $h=j \circ g: X^{*} \rightarrow \mathbb{R}^{n}$ is the $L^{*}$-biLipschitz embedding we were looking for.

Combining these lemmas, we have:

5.3. Lemma. For every $n \in \mathbb{N}^{*}$ and $c \geq 1$, there exists $L>1$ which satisfies the following condition: Let $(X, d)$ be a finite ultrametric space with $|X| \geq 2$ which can be L-bi-Lipschitz embedded in $\mathbb{R}^{n}$ and for which $\delta(X) \leq$ $c a(X)$. Then $|X| \leq 2 n$.

Proof. Let us fix $c \geq 1$. Obviously, we can choose $L_{n}>1$, for $n \in \mathbb{N}^{*}$, such that $L_{1}=\sqrt{1+1 /(2 c)}$ and $L_{n}=L_{n+1}\left(1-\left(L_{n+1}{ }^{4}-1\right)^{2} c^{4}\right)^{-1 / 4}$, for $n \in \mathbb{N}^{*}$. We are to prove that if $(X, d)$ is an ultrametric space with $|X| \geq 2$, $X$ is $L_{n}$-bi-Lipschitz embeddable in $\mathbb{R}^{n}$ and $\delta(X) \leq c a(X)$, then $|X| \leq 2 n$.

Assume for contradiction that there exists $m \in \mathbb{N}^{*}$, a finite ultrametric space $(X, d)$ with $|X|>2 m$ and $\delta(X) \leq c a(X)$, and an $L_{m}$-bi-Lipschitz embedding $f: X \rightarrow \mathbb{R}^{m}$. Suppose further that $m$ is the least possible. 
Lemma 5.1 implies that $m>1$, as $L_{1}<\sqrt{1+1 / c}$, so $m=n+1$, where $n \in \mathbb{N}^{*}$. Now $L_{n}$ and $L_{m}$ are so chosen that, by Lemma 5.2 , there are $x_{0}, x_{1} \in X$ and an $L_{n}$-bi-Lipschitz embedding $h: X^{*}=X \backslash\left\{x_{0}, x_{1}\right\} \rightarrow \mathbb{R}^{n}$. Because $\left|X^{*}\right|>2 n$ and $\delta\left(X^{*}\right) \leq \delta(X) \leq c a(X) \leq c a\left(X^{*}\right)$, we have a contradiction with the minimality of $m$.

As a by-product, we get the following well-known result [ABBW] about the isometric embeddability of ultrametric spaces. Of course, this corollary could also be shown directly in the manner that was outlined in the paragraph preceding Lemma 5.1.

5.4. Corollary. Suppose that $(X, d)$ is an ultrametric space which can be isometrically embedded in a Euclidean space $\mathbb{R}^{n}$ for some $n \in \mathbb{N}^{*}$. Then $X$ is finite.

Proof. Let us prove that for every $n \in \mathbb{N}^{*}$, if $(X, d)$ can be isometrically embedded in $\mathbb{R}^{n}$, then $|X| \leq 2 n$ (by [ABBW, Lemma 4.1], $n+1$ instead of $2 n$ is the best possible bound). Suppose this does not hold. Then there is an ultrametric space $(X, d)$ with $|X|=2 n+1$ and an isometric embedding $f: X \rightarrow \mathbb{R}^{n}$. Choose $c=\delta(X) / a(X)$ and note that $f$ is an $L$-bi-Lipschitz embedding for every $L>1$; then we have arrived at a contradiction with the previous lemma.

Having Lemma 5.3, it is quite easy to derive the theorem of this section. Although that lemma restricts to finite sets $X$ with bounded ratio $\delta(X) / a(X)$, the following proposition (cf. [A, Remarque 2]) shows that the estimate for $|X|$ in 5.3 implies a more general one.

5.5. Proposition. Let $(X, d)$ be a metric space, $k \in \mathbb{N}^{*}$ and $c>1$. Suppose that for every finite $Y \subset X$ with $|Y| \geq 2$, we know that if $Y \subset$ $B_{d}(x, c a(Y))$ for some $x \in Y$, then $|Y| \leq k$. Then $\operatorname{dim}_{\mathrm{A}}(X, d) \leq \log _{c} k$.

Proof. We prove by induction on $j \in \mathbb{N}^{*}$ that if $Y \in \mathcal{P}_{\omega}(X),|Y| \geq 2$ and $Y \subset B_{d}\left(x, c^{j} a(Y)\right)$ for some $x \in Y$, then $|Y| \leq k^{j}$. By hypothesis, the claim holds for $j=1$. Suppose now that the claim holds for $j \in \mathbb{N}^{*}$, and that $Y \in \mathcal{P}_{\omega}(X),|Y| \geq 2, x \in Y$ and $Y \subset B_{d}\left(x, c^{j+1} a(Y)\right)$. Let $Z \subset Y$ be a maximal set with the following two properties:

$1^{\circ} x \in Z$,

$2^{\circ}|Z| \leq 1$ or $a(Z) \geq c^{j} a(Y)$.

If $|Z| \geq 2$, we have $Z \subset Y \subset B_{d}\left(x, c^{j+1} a(Y)\right) \subset B_{d}(x, c a(Z))$; so in any case, $|Z| \leq k$. If $y \in Y$, then by the maximality of $Z$, there is $z \in Z$ such that $d(y, z)<c^{j} a(Y)$. For $z \in Z$, let $Y_{z}=\left\{y \in Y \mid d(y, z)<c^{j} a(Y)\right\}$; then either $\left|Y_{z}\right|=1$ or $a\left(Y_{z}\right) \geq a(Y)$ and thus $Y_{z} \subset B_{d}\left(z, c^{j} a\left(Y_{z}\right)\right)$, which implies $\left|Y_{z}\right| \leq k^{j}$, by the induction hypothesis. On the other hand, we have 
$Y \subset \bigcup_{z \in Z} Y_{z}$, so that $|Y| \leq \sum_{z \in Z}\left|Y_{z}\right| \leq k \cdot k^{j}=k^{j+1}$, and the induction is complete.

Let $s=\log _{c} k$. Assume $Y \subset X$ and $a, b \in \mathbb{R}_{+}^{*}$ are such that $a \leq b$ and for every distinct $x, y \in Y$, we have $a \leq d(x, y) \leq b$. Let $j=\left\lfloor\log _{c}(b / a)\right\rfloor+1 \in$ $\mathbb{N}^{*}$; then $c^{j} a>b$. I claim that $|Y| \leq k(b / a)^{s}$. We may assume that $|Y| \geq 2$ and $Y$ is finite. Then $a(Y) \geq a$ and for any $x \in Y, Y \subset B_{d}\left(x, c^{j} a\right) \subset$ $B_{d}\left(x, c^{j} a(Y)\right)$. Consequently, $|Y| \leq k^{j} \leq k(b / a)^{s}$. Hence, $(X, d)$ is $(k, s)$ homogeneous.

5.6. Theorem. For every $n \in \mathbb{N}^{*}$ and $\left.s \in\right] 0, n[$ there exists $L>1$ such that the following holds: If $(X, d)$ is an ultrametric space which can be $L$-bi-Lipschitz embedded in $\mathbb{R}^{n}$, then $\operatorname{dim}_{\mathrm{A}}(X, d) \leq s$.

Proof. Fix $n \in \mathbb{N}^{*}$ and $\left.s \in\right] 0, n\left[\right.$, let $c=(2 n)^{1 / s}>1$ and choose $L>1$ as in Lemma 5.3. Consider an ultrametric space $(X, d)$ which can be $L$-bi-Lipschitz embedded in $\mathbb{R}^{n}$ by some $f: X \rightarrow \mathbb{R}^{n}$. Let us apply Proposition 5.5 with $k=2 n$. Suppose that $Y \subset X$ is finite, $|Y| \geq 2$ and there is $x \in Y$ such that $Y \subset B_{d}(x, c a(Y))$. Since $d$ is an ultrametric, the last condition is actually equivalent to $\delta(Y)<c a(Y)$. Now we know that $f \nmid Y: Y \rightarrow \mathbb{R}^{n}$ is an $L$-bi-Lipschitz embedding, so by the choice of $L$, we get $|Y| \leq 2 n$. Hence, the hypothesis of Proposition 5.5 holds, and we get

$$
\operatorname{dim}_{\mathrm{A}}(X, d) \leq \log _{c} k=1 / \log _{k} k^{1 / s}=s .
$$

\section{References}

[ABBW] M. Aschbacher, P. Baldi, E. B. Baum and R. M. Wilson, Embeddings of ultrametric spaces in finite dimensional structures, SIAM J. Algebraic Discrete Methods 8 (1987), 564-577.

[A] P. Assouad, Étude d'une dimension métrique liée à la possibilité de plongements dans $\mathbb{R}^{n}$, C. R. Acad. Sci. Paris Sér. A 288 (1979), 731-734.

[LM-L] J. Luukkainen and H. Movahedi-Lankarani, Minimal bi-Lipschitz embedding dimension of ultrametric spaces, Fund. Math. 144 (1994), 181-193.

[M-LW] H. Movahedi-Lankarani and R. Wells, Ultrametrics and geometric measures, Proc. Amer. Math. Soc. 123 (1995), 2579-2584.

[S] S. Semmes, On the nonexistence of bilipschitz parameterizations and geometric problems about $A_{\infty}$ weights, Rev. Mat. Iberoamericana, to appear.

Department of Mathematics

University of Helsinki

P.O. Box 4 (Yliopistonkatu 5)

FIN-00014 Helsinki

Finland

E-mail: kerkko.luosto@helsinki.fi

Received 7 June 1995;

in revised form 9 January 1996 